\title{
A Preoperative Nomogram for Predicting Chemoresistance to Neoadjuvant Chemotherapy in Patients with Locally Advanced Cervical Squamous Carcinoma Treated with Radical Hysterectomy
}

\author{
Zhengjie Ou, Dan Zhao, Bin Li, Yating Wang, Shuanghuan Liu, Yanan Zhang \\ Department of Gynecology Oncology, National Cancer Center/National Clinical Research Center for Cancer/Cancer Hospital, \\ Chinese Academy of Medical Sciences and Peking Union Medical College, Beijing, China
}

\begin{abstract}
Purpose This study aimed to investigate the factors associated with chemoresistance to neoadjuvant chemotherapy (NACT) followed by radical hysterectomy $(\mathrm{RH})$ and construct a nomogram to predict the chemoresistance in patients with locally advanced cervical squamous carcinoma (LACSC).

Materials and Methods This retrospective study included 516 patients with International Federation of Gynecology and Obstetrics (2003) stage IB2 and IIA2 cervical cancer treated with NACT and RH between 2007 and 2017. Clinicopathologic data were collected, and patients were assigned to training $(n=381)$ and validation $(n=135)$ sets. Univariate and multivariate analyses were performed to analyze factors associated with chemoresistance to NACT. A nomogram was built using the multivariate logistic regression analysis results. We evaluated the discriminative ability and accuracy of the model using a concordance index and a calibration curve. The predictive probability of chemoresistance to NACT was defined as $>34 \%$.

Results Multivariate analysis confirmed menopausal status, clinical tumor diameter, serum squamous cell carcinoma antigen level, and parametrial invasion on magnetic resonance imaging before treatment as independent prognostic factors associated with chemoresistance to NACT. The concordance indices of the nomogram for training and validation sets were 0.861 ( $95 \%$ confidence interval [Cl], 0.822 to 0.900$)$ and $0.807(95 \% \mathrm{Cl}, 0.807$ to 0.888$)$, respectively. Calibration plots revealed a good fit between the modelpredicted probabilities and actual probabilities (Hosmer-Lemeshow test, $p=0.597$ ). Furthermore, grouping based on the nomogram was associated with progression-free survival.

Conclusion We developed a nomogram for predicting chemoresistance in LACSC patients treated with RH. This nomogram can help physicians make clinical decisions regarding primary management and postoperative follow-up of the patients.
\end{abstract}

Key words Uterine cervical neoplasms, Neoadjuvant chemotherapy, Chemoresistance, Nomograms

\section{Introduction}

Locally advanced cervical squamous carcinoma (LACSC) is recommended to be treated by two types of therapies, radical hysterectomy $(\mathrm{RH})$ and chemoradiotherapy [1]. Although several studies have revealed similar survival rates between these two treatment modalities, $\mathrm{RH}$ plays a unique and necessary role in the treatment of patients with LACSC due to the lack of radiotherapy devices in low-income districts [1-3]. Furthermore, neoadjuvant chemotherapy (NACT) combined with RH has a greater advantage over RH alone and is associated with a lower incidence of postoperative risk factors and a similar overall survival compared with other modalities [4], which prompt it a common and necessary treatment for LACSC in low-income districts. However, the overall response rate to NACT ranged from $56.5 \%$ to $92.0 \%[5,6]$, which means not all patients who underwent NACT could attain effective treatment outcomes, resulting in unnecessary therapy and delay of the best treatment opportunity. Thus, to optimize treatment efficiency, patients with chemoresistant characteristics should select a more appropriate treatment modality, such as concurrent chemoradiotherapy or $\mathrm{RH}$ alone, rather than NACT combined with RH. An effective and clinically validated approach that pre-therapeutically predicts chemotherapeutic resistance would thereby be of great benefit in avoiding superfluous chemotherapy before surgery, as well as treatment strategies and individualization and effective patient stratification.

Chemoresistance is an important factor in cervical cancer treatment and prognosis, whether it is derived from NACT or adjuvant chemotherapy. Previous studies have demonstrated that non-responders to NACT had lower survival and high-

\section{Correspondence: Dan Zhao}

Department of Gynecology Oncology, National Cancer Center/National Clinical Research Center for Cancer/Cancer Hospital, Chinese Academy of Medical Sciences and Peking Union Medical College, No.17, South Panjiayuan Residential, Chaoyang District, Beijing 100021, China

Tel: 86-010-87787384 Fax: 86-0971-6360700 E-mail: zhaodan@cicams.ac.cn

Received February 27, 2020 Accepted September 9, 2020 Published Online September 14, 2020 


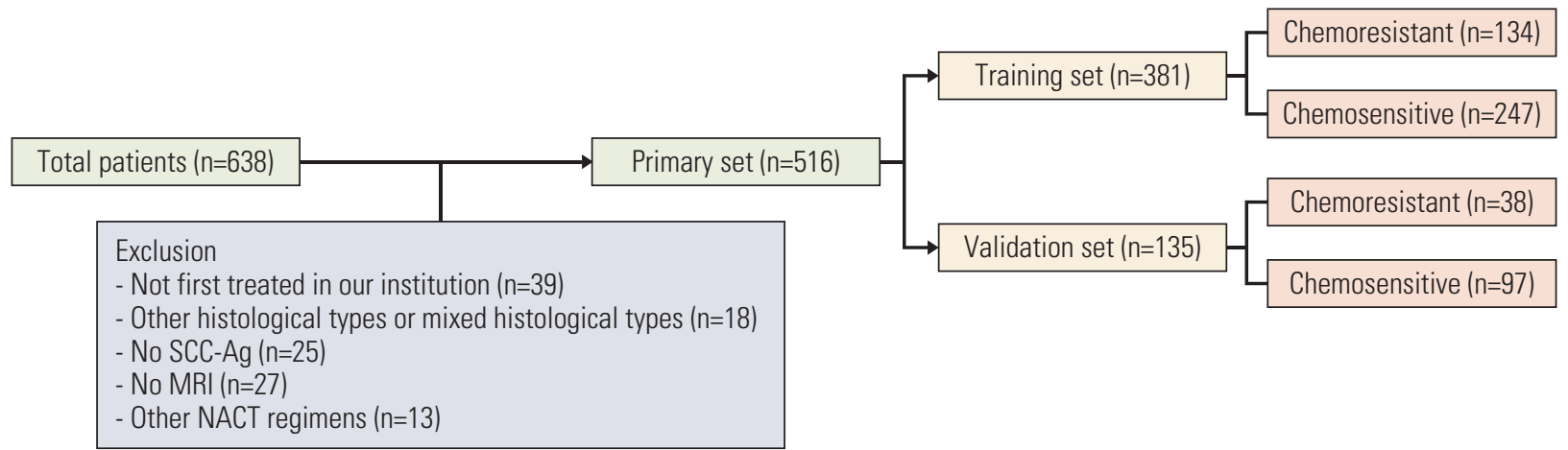

Fig. 1. Inclusion criteria. MRI, magnetic resonance imaging; NACT, neoadjuvant chemotherapy; SCC-Ag, squamous cell carcinoma antigen.

er recurrence rates than responders $(\mathrm{p}<0.001$ and $\mathrm{p}=0.013$, respectively) [7]. However, there has been no specific tumor marker or model derived from clinicopathologic factors that allow predicting chemoresistance accurately. Some studies suggested that the serum squamous cell carcinoma antigen (SCC-Ag) and lactate dehydrogenase may be associated with the overall response to NACT in cervical cancer [8,9], but there is still a lack of accuracy in prediction and explicit direction for the clinical doctor. Sun et al.'s study [10] made use of radiomic analysis to predict response to NACT, but it excluded clinicopathologic factors proven to be associated with chemosensitivity from their model. Other studies have only taken advantage of imaging material or clinicopathologic data to analyze the risk factors of chemoresistance to NACT, while few studies have constructed a concrete predictive model. Thus, combining clinicopathological and imaging material is crucial to building an effective and available model for clinicians.

This study aimed to investigate the risk factors associated with chemoresistance to NACT following RH and construct a nomogram model based on analysis of pretreatment data to predict chemoresistance to NACT in patients with LACSC.

\section{Materials and Methods}

\section{Patients}

We retrospectively collected clinicopathologic data of 638 patients with LACSC treated with NACT and nerve-sparing RH with retroperitoneal lymphadenectomy between August 2007 and August 2013 in our institution (National Cancer Center/Cancer Hospital, Chinese Academy of Medical Sciences; CICAMS). The inclusion criteria (Fig. 1) were as follows: patients who were diagnosed with cervical squamous cell carcinoma on cervical biopsy; patients with International Federation of Gynecology and Obstetrics (FIGO, 2003) stage
IB2 and IIA2 cervical squamous carcinoma; patients with cervical squamous carcinoma firstly treated in our institution; patients treated with combined chemotherapy of platinum and paclitaxel before surgery; patients without severe complications who could tolerate chemotherapy and surgery and those who had never received chemotherapy, radiotherapy, or immune therapy; and patients with complete clinicopathologic data. We excluded 39 patients who had been treated with chemotherapy in other institutions, 18 patients with other histological types or mixed histological types in terms of the pathological exam, 25 patients whose tumor biomarkers had not been measured, 27 patients who did not undergo pelvic magnetic resonance imaging (MRI) before and after NACT, and 13 patients treated with other NACT regimens. Thus, a total of 516 patients were enrolled in this study. This study was approved by the center's institutional review board.

The clinical tumor diameter was defined as the maximum tumor diameter measured on MRI. The diameter-based tumor volume on MRI was derived from the formula of the ellipsoid volume $\mathrm{d} 1 \times \mathrm{d} 2 \times \mathrm{d} 3 \times \pi / 6$ [11]. The longitudinal diameter (d1) along the long axis of the uterine cavity was measured in the sagittal plane, the anteroposterior diameter (d2) was measured as the diameter perpendicular to the longitudinal diameter (d1) in the sagittal plane, and the largest lateral diameter $(\mathrm{d} 3)$ was measured in the axial plane. Specifically, parametrial invasion on MRI was defined as a full or partial loss of normal low-signal-intensity cervical stromal ring on T2-weighed MRI. Lymphadenectasis on MRI was defined as the occurrence of an enlarged lymph node of $>5$ $\mathrm{mm}$ on pelvic MRI.

The response of NACT was assessed by the change of tumor volume on MRI according to the Response Evaluation Criteria in Solid Tumors (RECIST v.1.1). The clinical response was evaluated before surgery and classified as follows: CR, complete response; $\mathrm{PR}$, partial response; $\mathrm{SD}$, stable disease; 
Table 1. Preoperative clinicopathological factors in training set and validation set

\begin{tabular}{|c|c|c|c|}
\hline Characteristic & Training set $(\mathrm{n}=381)$ & Validation set $(n=135)$ & p-value \\
\hline Age (yr) & $45.0(22.0-65.0)$ & $46.0(16.0-66.0)$ & 0.443 \\
\hline BMI $\left(\mathrm{kg} / \mathrm{m}^{2}\right)$ & $24.0 \pm 3.7$ & $23.9 \pm 3.4$ & 0.795 \\
\hline \multicolumn{4}{|l|}{ Menopausal status } \\
\hline No & $321(84.3)$ & $110(81.5)$ & 0.456 \\
\hline Yes & $60(15.7)$ & $25(18.5)$ & \\
\hline \multicolumn{4}{|l|}{ FIGO stage (2003) } \\
\hline IB2 & $268(70.3)$ & $87(64.4)$ & 0.204 \\
\hline IIA2 & $113(29.7)$ & $48(35.6)$ & \\
\hline \multicolumn{4}{|l|}{ Grossly type } \\
\hline Exophytic & $348(91.3)$ & $130(96.3)$ & 0.058 \\
\hline Endophytic & $33(8.7)$ & $5(3.7)$ & \\
\hline \multicolumn{4}{|l|}{ Grade } \\
\hline Good & $22(5.8)$ & $9(6.7)$ & 0.719 \\
\hline Moderate & $99(26.0)$ & $39(28.9)$ & \\
\hline Poor & $260(68.2)$ & $87(64.4)$ & \\
\hline Clinical tumor diameter $(\mathrm{cm})$ & $5.0(4.0-8.0)$ & $5.0(4.0-8.0)$ & 0.908 \\
\hline Serum SCC-Ag level (ng/mL) & $3.9(0.1-152.5)$ & $3.8(0.3-160.1)$ & 0.315 \\
\hline Tumor volume on MRI $\left(\mathrm{cm}^{3}\right)$ & $39.3(1.6-320.8)$ & $33.7(3.6-179.6)$ & 0.724 \\
\hline \multicolumn{4}{|c|}{ Parametrial invasion on MRI before treatment } \\
\hline Negative & $285(74.8)$ & $99(73.3)$ & 0.737 \\
\hline Positive & $96(25.2)$ & $36(26.7)$ & \\
\hline \multicolumn{4}{|c|}{ Lymphadenectasis on MRI before treatment } \\
\hline Negative & $198(52.0)$ & $72(53.3)$ & 0.785 \\
\hline Positive & $183(48.0)$ & $63(46.7)$ & \\
\hline \multicolumn{4}{|l|}{ NACT regimen } \\
\hline $\mathrm{TP}$ & $143(37.5)$ & $40(29.6)$ & 0.255 \\
\hline $\mathrm{TC}$ & $84(22.0)$ & $33(24.4)$ & \\
\hline TN & $154(40.4)$ & $62(45.9)$ & \\
\hline \multicolumn{4}{|l|}{ NACT cycle } \\
\hline 1 & $95(24.9)$ & $28(20.7)$ & 0.326 \\
\hline$\geq 2$ & $286(75.1)$ & $107(79.3)$ & \\
\hline \multicolumn{4}{|l|}{ Chemosensitivity } \\
\hline Resistant & $134(35.2)$ & $38(28.1)$ & 0.137 \\
\hline Sensitive & $247(64.8)$ & 97 (71.9) & \\
\hline
\end{tabular}

Values are presented as median (range), mean \pm SD, or number (\%). BMI, body mass index; FIGO, International Federation of Gynecology and Obstetrics; MRI, magnetic resonance imaging; NACT, neoadjuvant chemotherapy; SCC-Ag, squamous cell carcinoma antigen; SD, standard deviation; TC, paclitaxel plus carboplatin; TN, paclitaxel plus nedaplatin; TP, paclitaxel plus cisplatin.

and $\mathrm{PD}$, progressive disease [12]. Patients with CR or PR (responders) were assigned to the chemosensitive group, while patients with SD or PD (non-responders) were assigned to the chemoresistant group.

\section{NACT regimens}

The NACT regimens in this study consisted of chemotherapies combining paclitaxel and platinum, including paclitaxel and cisplatin (TP), paclitaxel, and carboplatin (TC), and paclitaxel and nedaplatin (TN). All enrolled patients received 1-3 cycles of NACT intravenously every 21 days.
The cycles of NACT were based on the situation of the patients and the effect of chemotherapy, as assessed by the physician.

\section{Statistical analysis}

The training and validation sets were distributed in the ratio of 7:3 from the primary set. Preoperative clinicopathologic factors including serum SCC-Ag, parametrial invasion on MRI, and lymphadenectasis on MRI were compared between the chemosensitive and chemoresistant groups. Data distribution was evaluated using the Kolmogorov- 
Table 2. Comparison of preoperative clinicopathologic factors between chemoresistant and chemosensitive patients in training set

\begin{tabular}{|c|c|c|c|}
\hline Factor & Chemoresistant ( $\mathrm{n}=134)$ & Chemosensitive $(\mathrm{n}=247)$ & p-value \\
\hline Age (yr) & $43.0(25.0-60.0)$ & $45.0(22.0-65.0)$ & 0.019 \\
\hline BMI (kg/m²) & $23.6 \pm 3.7$ & $24.2 \pm 3.7$ & 0.122 \\
\hline \multicolumn{4}{|l|}{ Menopausal status } \\
\hline No & $121(37.7)$ & $200(62.3)$ & 0.017 \\
\hline Yes & $13(21.7)$ & $47(78.3)$ & \\
\hline \multicolumn{4}{|l|}{ FIGO stage (2003) } \\
\hline IB2 & $94(35.1)$ & $174(64.9)$ & 0.952 \\
\hline IIA2 & $40(35.4)$ & $73(64.6)$ & \\
\hline \multicolumn{4}{|l|}{ Grossly type } \\
\hline Exophytic & $121(34.8)$ & $227(65.2)$ & 0.595 \\
\hline Endophytic & $13(39.4)$ & $20(60.6)$ & \\
\hline \multicolumn{4}{|l|}{ Grade } \\
\hline Good & $7(31.8)$ & $15(68.2)$ & 0.838 \\
\hline Moderate & $37(37.4)$ & $62(62.6)$ & \\
\hline Poor & $90(34.6)$ & $180(65.4)$ & \\
\hline Clinical tumor diameter $(\mathrm{cm})$ & $4.5(4.0-7.2)$ & $5.0(4.0-8.0)$ & $<0.001$ \\
\hline Serum SCC-Ag level (ng/mL) & $1.5(0.3-26.1)$ & $6.2(0.1-46.2)$ & $<0.001$ \\
\hline Tumor volume on MRI (cm $\left.{ }^{3}\right)$ & $46.1(2.6-179.6)$ & $36.1(1.6-320.8)$ & 0.123 \\
\hline \multicolumn{4}{|c|}{ Parametrial invasion on MRI before treatment } \\
\hline Negative & $75(26.3)$ & $210(73.7)$ & $<0.001$ \\
\hline Positive & $59(61.5)$ & $37(38.5)$ & \\
\hline \multicolumn{4}{|c|}{ Lymphadenectasis on MRI before treatment } \\
\hline Negative & $66(33.3)$ & $132(66.7)$ & 0.435 \\
\hline Positive & $68(37.2)$ & $115(62.8)$ & \\
\hline \multicolumn{4}{|l|}{ NACT regimens } \\
\hline $\mathrm{TP}$ & $58(40.6)$ & $85(59.4)$ & 0.192 \\
\hline TC & $29(34.5)$ & $55(65.5)$ & \\
\hline $\mathrm{TN}$ & $47(30.5)$ & $107(69.5)$ & \\
\hline \multicolumn{4}{|l|}{ NACT cycle } \\
\hline 1 & $38(40.0)$ & $57(60.0)$ & 0.255 \\
\hline$\geq 2$ & $96(33.6)$ & $190(66.4)$ & \\
\hline
\end{tabular}

Values are presented as median (range), mean \pm SD, or number (\%). BMI, body mass index; FIGO, International Federation of Gynecology and Obstetrics; MRI, magnetic resonance imaging; NACT, neoadjuvant chemotherapy; SCC-Ag, squamous cell carcinoma antigen; SD, standard deviation; TC, paclitaxel plus carboplatin; TN, paclitaxel plus nedaplatin; TP, paclitaxel plus cisplatin.

Smirnov normality test. Continuous variables were analyzed using Student's $\mathrm{t}$ test or the Mann-Whitney $U$ test according to the data distribution, while categorical variables were compared between the two groups using the Pearson chi-square test or Fisher exact test. The clinicopathologic factors for sensitivity to NACT were determined based on multivariate logistic regression analysis using a backward elimination method. Data were analyzed using SPSS ver. 25.0 (IBM Corp., Armonk, NY). A nomogram for chemoresistance was constructed based on the results of the binary logistic regression model using the rms package in $\mathrm{R}$ ver. 3.6.1 (https://www.r-project.org/), as described previously [13]. A concordance index and a calibration curve were used to evaluate the accuracy and discrimination of the model for the training and validation sets, which were derived based on the regression analysis. The calibration of the model was assessed using the Hosmer-Lemeshow test. The chemosensitive group was predefined as having a predicted probability of chemoresistance $<34 \%$. Furthermore, the overall survival curves for the chemoresistant and chemosensitive groups were formed using the Kaplan-Meier method and log-rank test. A p-value (two-sided) of $<0.05$ was considered as statistically significant. 
Table 3. Multivariate analysis of preoperative clinicopathologic factors for predicting chemoresistance of NACT

\begin{tabular}{lcc} 
Variable & $\begin{array}{c}\text { Multivariate analysis } \\
\text { odds ratio (95\% CI) }\end{array}$ & p-value \\
\hline Menopausal status & $0.210(0.088-0.503)$ & $<0.001$ \\
\hline $\begin{array}{l}\text { Parametrial invasion on } \\
\text { MRI before treatment }\end{array}$ & $9.148(4.805-17.417)$ & $<0.001$ \\
Clinical tumor diameter $(\mathrm{cm})$ & $0.388(0.267-0.563)$ & $<0.001$ \\
Serum SCC-Ag level $(\mathrm{ng} / \mathrm{mL})$ & $0.847(0.796-0.900)$ & $<0.001$ \\
\hline
\end{tabular}

$\mathrm{CI}$, confidence interval; MRI, magnetic resonance imaging; NACT, neoadjuvant chemotherapy; SCC-Ag, squamous cell carcinoma antigen.

\section{Results}

\section{General characteristics of the patients}

Among the 516 patients enrolled in this study, 381 were assigned to the training set and 135 to the validation set (Table 1, Fig. 1). Of the 381 subjects in the training set, 247 $(64.8 \%)$ were chemosensitive patients, including $27(7.1 \%)$ with CR and 220 (57.7\%) with PR, and 134 (35.2\%) were chemoresistant patients. No subjects showed PD after NACT. The median age at diagnosis was 45 years (range, 22 to 65 years). FIGO stage was distributed as follows: 268 patients with stage IB2 (70.3\%) and 113 with stage IIA2 (29.7\%). All subjects in the training set were divided into two groups in terms of sensitivity to NACT.

\section{Predictors of chemoresistance to NACT in squamous cell carcinoma}

In the univariate analysis (Table 2), chemoresistance was not significantly associated with body mass index, FIGO stage, grade, gross type, tumor volume on MRI, and lymphadenectasis on MRI before treatment or the NACT cycle $(p>0.05)$. Chemoresistance was, however, associated with age ( 43 years vs. 45 years, $\mathrm{p}=0.019$ ), menopausal status (premenopause, $23.8 \%$, vs. postmenopause, $7.1 \%$; $p=0.017$ ), parametrial invasion on MRI before treatment (positive, $61.5 \%$, vs. negative, $26.3 \% ; \mathrm{p}<0.001)$, clinical tumor diameter $(4.5$ $\mathrm{cm}$ vs. $5.0 \mathrm{~cm}, \mathrm{p}<0.001)$, and pretreatment SCC-Ag level (1.5 $\mathrm{ng} / \mathrm{mL}$ vs. $6.2 \mathrm{ng} / \mathrm{mL}, \mathrm{p}<0.001)$.

The multivariate analysis (Table 3) confirmed menopausal status (odds ratio [OR], 0.210; 95\% confidence interval [CI], 0.088 to $0.503 ; \mathrm{p}<0.001$ ), parametrial invasion on MRI before treatment (OR, 9.148; 95\% CI, 4.805 to 17.417; $\mathrm{p}<$ 0.001 ), clinical tumor diameter (OR, 0.388 ; $95 \%$ CI, 0.267 to 0.563; $\mathrm{p}<0.001)$, and serum SCC-Ag level (OR, $0.847 ; 95 \% \mathrm{CI}$, 0.796 to $0.900 ; p<0.001)$ as independent factors associated with chemoresistance to NACT.

\section{Nomogram construction and verification}

The variables used in constructing the nomogram were based on the multivariate analysis results (Fig. 2). A point value proportional to the $\mathrm{OR}$ was assigned to each variable. For instance, parametrial invasion on MRI in this model was given 27 points, while clinical tumor diameter was given 11.4 points per centimeter. The $\mathrm{p}$-value of the Hosmer-Lemeshow test for the predictive model was 0.597 , which indicates a good fit. The concordance index of the model for the training set was 0.861 (95\% CI, 0.822 to 0.900 ), while that for the validation set was 0.807 (95\% CI, 0.807 to 0.888), as shown in Fig. $3 \mathrm{~A}$ and B. In Fig. $3 \mathrm{C}$ and D, the thick solid line (performance of the model) is positioned close to the thin solid line (performance of an ideal model) in both the training and validation sets. Thus, the calibration plots revealed a good agreement

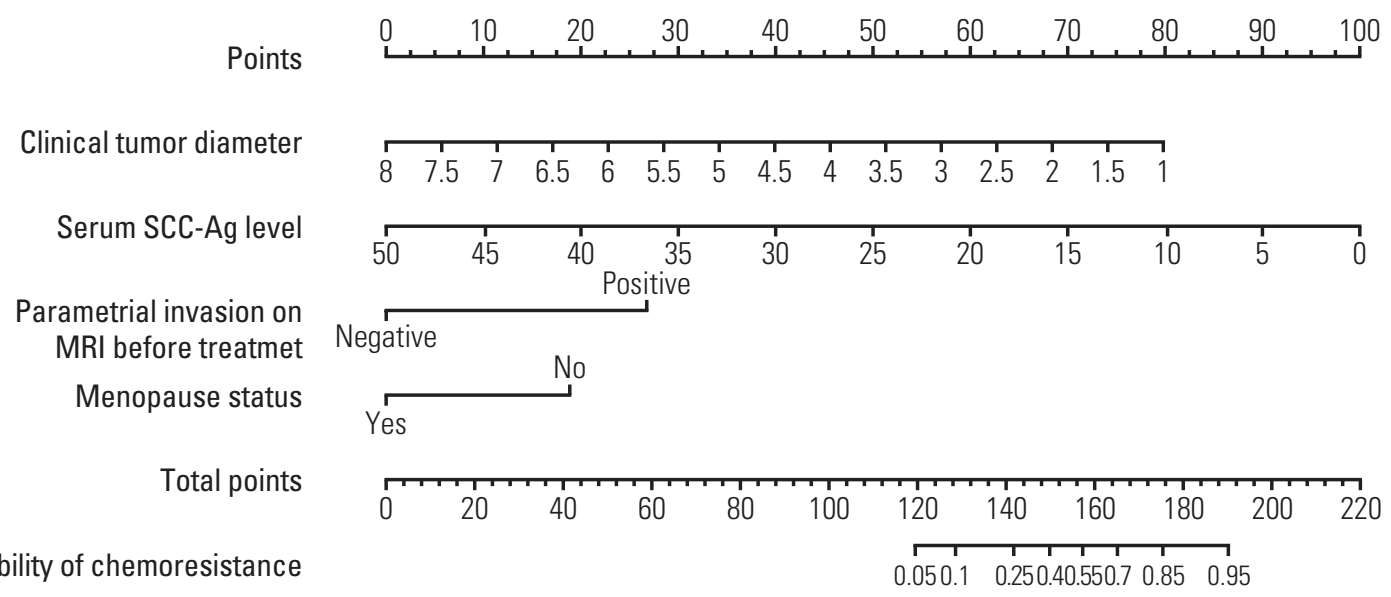

Fig. 2. Nomogram predicting chemoresistance in patients with locally advanced cervical squamous carcinoma treated with neoadjuvant chemotherapy and radical hysterectomy. MRI, magnetic resonance imaging; SCC-Ag, squamous cell carcinoma antigen. 

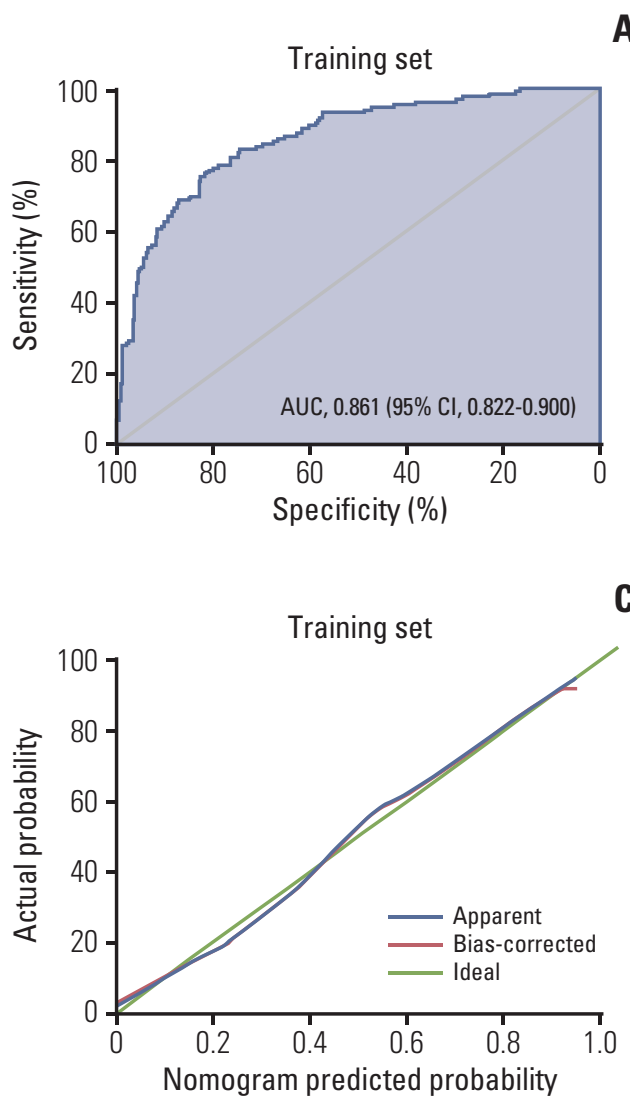

A
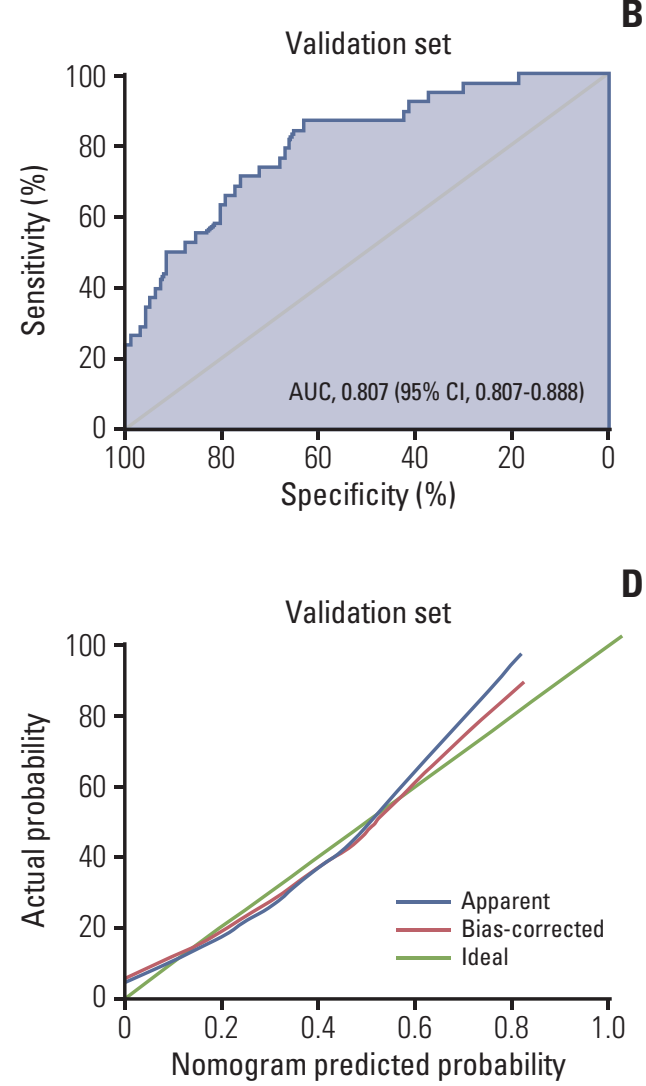

Fig. 3. Receiver operating characteristic curve for prediction of chemoresistance based on nomogram model in training set (A) and validation set (B). Calibration plot for nomogram model in training set (C) and validation set (D). Green line, the ideal reference line, indicated the perfect prediction of ideal model; Blue line, the apparent line, represented the entire cohort in training set or validation set; Red line, the bias-corrected line, is bias-corrected by bootstrapping $(B=1,000$ repetitions), suggesting observed performance of current nomogram. AUC, area under curve; $\mathrm{CI}$, confidence interval.

between the observed probabilities and nomogram-predicted probabilities in the training and validation sets.

\section{Chemosensitivity grouping according to the nomogram model}

Based on the receiver operating characteristic (ROC) curve in the training set, the Youden index was 0.497, while the probability of chemoresistance was $34 \%$. Therefore, $34 \%$ was defined as the cutoff for the risk ranking of chemoresistance. Table 4 shows the predicted and actual probabilities in terms of risk ranking for the validation set. In the validation set, the nomogram classified 51 of 135 patients as chemoresistant and 84 patients as chemosensitive. In the chemoresistant group, the predicted probability of chemoresistance was $51.7 \%$ and the actual chemoresistance rate was $52.9 \%$ (27 of 51 patients). In the chemosensitive group (84 of 135 patients), the predicted probability of chemoresistance was $13.8 \%$ and the actual chemoresistance rate was $13.1 \%$ (11 of 84 patients). To further test the validity of the grouping derived from the nomogram model, the survival curve was compared between the chemosensitive and chemoresistant groups. Finally, the progression-free survival (PFS) analysis revealed a significant difference between the two groups of the primary set ( $\mathrm{p}=0.022$ ) (Fig. 4).

\section{Discussion}

To assess the effect of NACT before treatment, we selected several clinicopathologic factors and characteristics on MRI to construct a nomogram that will predict chemoresistance to NACT in LACSC patients with high accuracy. To the best of our knowledge, no studies have yet undertaken this approach. Moreover, our analysis revealed several risk factors that were significantly associated with chemoresistance, including menopausal status and parametrial invasion on MRI, which have not been detected in previous studies. The chemosensitivity grouping based on a nomogram may have 
Table 4. Predicted probability and actual chemoresistant rate according to group depending on chemosensitivity

\begin{tabular}{lcc} 
& $\begin{array}{c}\text { Chemoresistant } \\
\text { group }\end{array}$ & $\begin{array}{c}\text { Chemosensitive } \\
\text { group }\end{array}$ \\
\hline $\begin{array}{c}\text { Predefined predicted } \\
\text { probability (\%) }\end{array}$ & $\geq 34$ & $<34$ \\
Total score (points) & $\geq 130$ & $<130$ \\
No. of patients & 51 & 84 \\
Predicted probability $(\%)$ & 51.72 & 13.84 \\
Actual rate, $\mathrm{n}(\%)$ & $27 / 51(52.9)$ & $11 / 84(13.1)$ \\
\hline
\end{tabular}

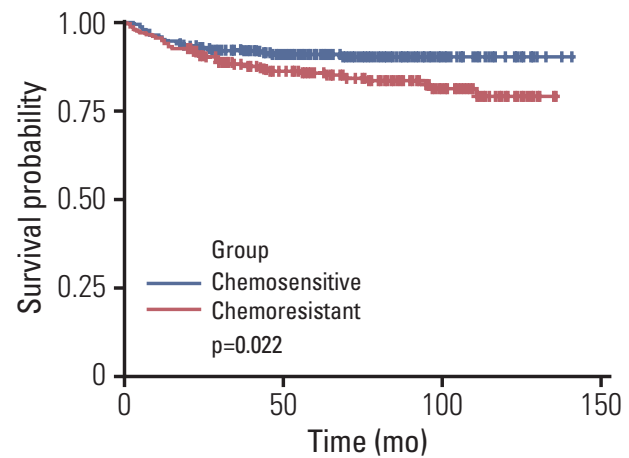

Fig. 4. Progression-free survival plot based on grouping of nomogram model for chemoresistance.

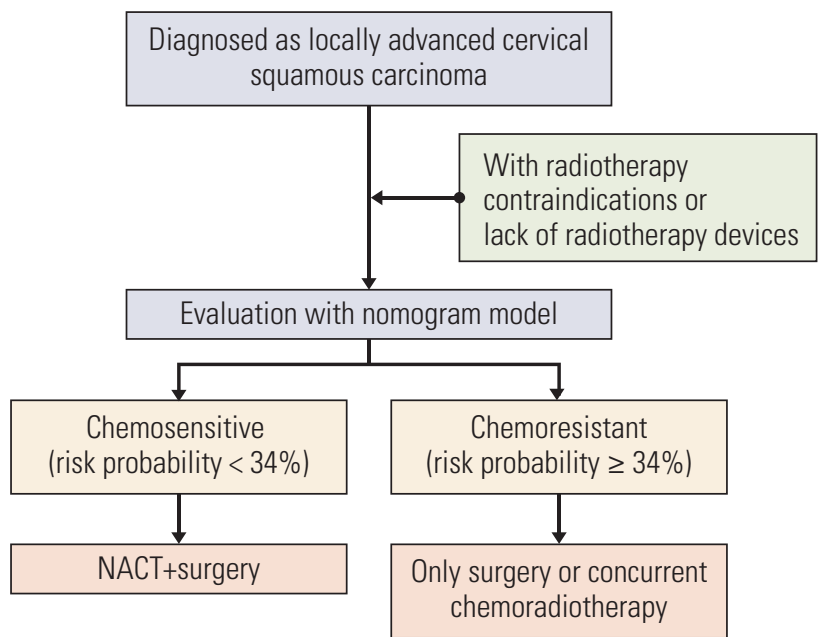

Fig. 5. Treatment-flow chart based on chemoresistance. NACT, neoadjuvant chemotherapy.

potential implications in the individual treatment strategy and prognosis of locally advanced cervical cancer.

Nomograms have been used to predict several clinicopathological factors such as pelvic lymph node metastasis, lymph-vascular space invasion, with high practicality and accuracy. A nomogram can integrate specific disease-associated factors, some of which may have been newly found in recent research, into a model to promote predictive performance. With a direct view and digitized result, it is convenient for application and individual stratification. A model based on MRI material had been proven to achieve high accuracy in judging the response of NACT via radiomic analysis in Sun's study [10]. A clinician may not be able to analyze patient data the way it is done in machine learning combining complex and meticulous imaging data. In our study, we selected several radiologic characteristics of clinical significance for our analysis, including tumor volume, parametrial invasion, and lymphadenectasis on MRI, which could be crucial for the diagnosis and prognosis of cervical cancer. Furthermore, certain clinicopathological factors before treatment have been confirmed to be related to the response of NACT, such as SCC-Ag, numbers of NACT cycles, and tumor size [6,14]. Wang et al.'s study [15] has found that integrating radiomic signature and clinicopathological factors were more efficient than the clinical model in predicting the pelvic lymph node metastasis. To improve the efficiency and accuracy, our study integrated imaging signature and clinicopathological data into model building.

Menopausal status is associated with age. Previous studies have also indicated that age is correlated with response to NACT and chemoradiotherapy in cervical cancer, while these studies have not included menopausal status in their analysis $[6,16]$. In this study, the univariate analysis showed that menopause and age were both associated with chemosensitivity, but the multivariate analysis only revealed menopause as a risk factor of chemoresistance. These results indicated that the association between chemosensitivity and age was partially based on menopausal status. Moreover, menopausal status plays a more important role than age does in the prediction of chemoresistance. Besides, some studies in breast cancer have revealed that menopausal status was considered as a predictor of pathological response to NACT; however, there is no research addressing such correlation in cervical cancer patients $[17,18]$. Moreover, similar to our study, it was shown before that premenopausal status is correlated with non-response of NACT. Premenopausal patients had lower levels of tumor biomarkers [19], consistent with the findings presented in our study. Lower tumor biomarker is correlated with lower pathological and clinical response to NACT in breast cancer [20], while lower SCCAg level was a risk factor of chemoresistance in our study, which suggests that menopausal status can be a significant predictor for chemoresistance. Moreover, a previous study has demonstrated that progenitor cells, which are fewer in postmenopausal patients, were resistant to adjuvant chemotherapy due to their active cell cycle, which may indicate that 
premenopausal status is correlated with chemoresistance [21]. Further studies are needed to confirm the correlation between chemosensitivity and menopausal status.

Integrating imaging data prominently improves the performance of a model. While tumor volume on MRI and lymphadenectasis have a lower predictive effect, parametrial invasion on MRI plays an important role in predicting chemoresistance. Some studies have indicated an essential MRI-surgical correlation, and the evaluation of tumors on MRI was more accurate than that on CT or by physical examinations in cervical cancer [22]. Meanwhile, parametrial invasion is important in cervical cancer staging and adjuvant radiotherapy. Poujade et al.'s study [16] has suggested an association between parametrial invasion and response to concurrent chemoradiotherapy before surgery $(p<0.001)$. Park et al.'s study [23] has also reported that parametrial invasion on postoperative pathology is associated with the response to NACT ( $\mathrm{p}=0.003)$. Our study has confirmed the correlation between parametrial invasion on MRI before treatment and chemoresistance. Cervical cancer with parametrial invasion may possess more invasive tumor cells. Invasive tumor cells tend to be exposed to intensive genetic stress leading to genetic instability and induction of drug resistance through various mechanisms due to hypoxic conditions [24]. Invasiveness into neighboring tissue contributes to drug resistance due to the existence of cancer stem cells among invasive tumor cells [25]. All of the above suggested that concurrent chemoradiotherapy rather than NACT $+\mathrm{RH}$ should be a preferred treatment in such patients with parametrial invasion on MRI because of the resistance to NACT.

Controversies exist between tumor diameter and SCC-Ag level and chemoresistance. Previous studies have reported that larger tumor diameter and SCC-Ag level might be correlated with chemoresistance to NACT, contrary to our study findings $[6,14]$. There are several reasons for this controversy. First, these studies included other histological types of cervical cancers besides squamous carcinoma, while our study only included patients with squamous carcinoma. Furthermore, these two factors have been proven to be positively associated with the appearance of several clinical factors, including pelvic lymphatic metastasis and uterine corpus tumor invasion, and even worse prognosis in previous studies [26]. In other words, larger tumor diameter and higher serum SCC-Ag level reflect faster growth and heavier progression of cervical cancer. Chemotherapy regimens, paclitaxel and platinum, as cytotoxic agents, are more likely to kill mitotic tumor cells, which is more likely to happen in neoplasms with a high degree of progression $[27,28]$. Moreover, previous studies suggested that the reduction rate of SCC-Ag before and after treatment is positively correlated with the response to treatment [9]. However, the lower pretreatment
SCC-Ag level is associated with a lower reducible degree after treatment, which is probably an indicator of chemoresistance. In our study, based on the negative correlation between the reduction rate of SCC-Ag level and chemoresistance, the patients with a high reduction rate tended to have a higher SCC-Ag level and larger tumor diameter before treatment. Therefore, it is reasonable that tumor diameter and serum SCC-Ag level could contribute effectively to a model's predictive capacity of chemoresistance to NACT.

Based on the abovementioned risk factors, we constructed a nomogram model to predict chemoresistance to NACT. We classified LACSC patients into two groups, chemoresistant and chemosensitive groups, according to the Youden index from the ROC curve of the nomogram to exclude patients unsuitable for NACT. Some studies have demonstrated that a good response is correlated with longer survival in cervical cancer [29]. As shown in Fig. 4, the model-based grouping can be significantly differentiated in terms of PFS, with the chemoresistant group exhibiting a worse prognosis than the chemosensitive group. This model-based grouping can also be utilized in clinical practice. As depicted in Fig. 5, regulations may be designed based on the nomogram model for LACSC patients with radiotherapy contraindications and the ones in areas, where radiotherapy devices are scare. If a patient is diagnosed with LACSC, she can be evaluated using the nomogram to confirm eligibility for NACT. Furthermore, it would convenient to put up an online nomogram with high accuracy to enable clinicians to assess patients' conditions remotely.

This study has some limitations. First, the study included only patients treated in a single institution, which may have caused selection bias. Second, the accuracy of the model could not reach $100 \%$ through validation. Third, due to constraints, the model could not be validated by an external data set. Despite these limitations, the current study has firstly built a nomogram model and evaluated it in a large number of patients with LACSC with respect to predicting chemoresistance to NACT. This study is definitely valuable for making individualized optimal therapy decisions that may help in avoiding ineffective treatment. With the deepening of research on chemosensitivity and the emergence of biomarkers associated with chemosensitivity, more promising models based on clinicopathologic factors, radiologic characteristics, and results of gene sequencing are expected.

In conclusion, this study developed and validated a nomogram model combining pretreatment factors to predict chemoresistance to NACT in patients with LACSC who were treated with RH. The model and the model-based grouping can help clinicians assess the probability of chemosensitivity to NACT and decide on the appropriate therapy for LACSC patients before surgery, especially in areas with limited 
access to radiotherapy devices.

\section{Ethical Statement}

The study was approved by the Ethics Committee of National Cancer Center/Cancer Hospital, Chinese Academy of Medical Sciences (Beijing, China). Written informed consent was obtained from all patients.

\section{Author Contributions}

Conceived and designed the analysis: Ou Z, Zhao D.

Collected the data: Ou Z, Wang Y, Liu S, Zhang Y.

Contributed data or analysis tools: Li B.
Performed the analysis: Ou Z.

Wrote the paper: Ou Z, Zhao D.

\section{Conflicts of Interest}

Conflicts of interest relevant to this article was not reported.

\section{Acknowledgments}

This study was supported by National Natural Science Foundation of China (81802619), the special fund for China Cancer Foundation Beijing Hope Marathon (LC2018A13), and CAMS Initiative for Innovative Medicine (No. 2017-I2M-2-003).

\section{References}

1. Bhatla N, Aoki D, Sharma DN, Sankaranarayanan R. Cancer of the cervix uteri. Int J Gynaecol Obstet. 2018;143 Suppl 2:2236.

2. He D, Duan C, Chen J, Lai L, Chen J, Chen D. The safety and efficacy of the preoperative neoadjuvant chemotherapy for patients with cervical cancer: a systematic review and meta analysis. Int J Clin Exp Med. 2015;8:14693-700.

3. Neoadjuvant Chemotherapy for Locally Advanced Cervical Cancer Meta-analysis Collaboration. Neoadjuvant chemotherapy for locally advanced cervical cancer: a systematic review and meta-analysis of individual patient data from 21 randomised trials. Eur J Cancer. 2003;39:2470-86.

4. Hsieh HY, Huang JW, Lu CH, Lin JC, Wang L. Definite chemoradiotherapy is a competent treatment option in FIGO stage IB2 cervical cancer compared with radical surgery +/- neoadjuvant chemotherapy. J Formos Med Assoc. 2019;118:99-108.

5. Katsumata N, Yoshikawa H, Kobayashi H, Saito T, Kuzuya $\mathrm{K}$, Nakanishi T, et al. Phase III randomised controlled trial of neoadjuvant chemotherapy plus radical surgery vs radical surgery alone for stages IB2, IIA2, and IIB cervical cancer: a Japan Clinical Oncology Group trial (JCOG 0102). Br J Cancer. 2013;108:1957-63.

6. Marita A, Ordeanu C, Rancea A, Nicolae T, Nagy VM. Longterm survival following neoadjuvant chemotherapy and concomitant radiochemotherapy in locally advanced cervical cancer: results of the Oncology Institute "Prof. Dr. Ion Chiricuta" experience. J Med Life. 2018;11:42-50.

7. Chen H, Liang C, Zhang L, Huang S, Wu X. Clinical efficacy of modified preoperative neoadjuvant chemotherapy in the treatment of locally advanced (stage IB2 to IIB) cervical cancer: randomized study. Gynecol Oncol. 2008;110:308-15.

8. Li J, Wu MF, Lu HW, Chen Q, Lin ZQ, Wang LJ. Pretreatment serum lactate dehydrogenase is an independent prognostic factor for patients receiving neoadjuvant chemotherapy for locally advanced cervical cancer. Cancer Med. 2016;5:1863-72.

9. Yin M, Hou Y, Zhang T, Cui C, Zhou X, Sun F, et al. Evaluation of chemotherapy response with serum squamous cell carci- noma antigen level in cervical cancer patients: a prospective cohort study. PLoS One. 2013;8:e54969.

10. Sun C, Tian X, Liu Z, Li W, Li P, Chen J, et al. Radiomic analysis for pretreatment prediction of response to neoadjuvant chemotherapy in locally advanced cervical cancer: a multicentre study. EBioMedicine. 2019;46:160-9.

11. Mayr NA, Taoka T, Yuh WT, Denning LM, Zhen WK, Paulino $\mathrm{AC}$, et al. Method and timing of tumor volume measurement for outcome prediction in cervical cancer using magnetic resonance imaging. Int J Radiat Oncol Biol Phys. 2002;52:14-22.

12. Eisenhauer EA, Therasse P, Bogaerts J, Schwartz LH, Sargent D, Ford R, et al. New response evaluation criteria in solid tumours: revised RECIST guideline (version 1.1). Eur J Cancer. 2009;45:228-47.

13. Iasonos A, Schrag D, Raj GV, Panageas KS. How to build and interpret a nomogram for cancer prognosis. J Clin Oncol. 2008;26:1364-70.

14. Scambia G, Benedetti Panici P, Foti E, Amoroso M, Salerno G, Ferrandina $G$, et al. Squamous cell carcinoma antigen: prognostic significance and role in the monitoring of neoadjuvant chemotherapy response in cervical cancer. J Clin Oncol. 1994;12:2309-16.

15. Wang T, Gao T, Yang J, Yan X, Wang Y, Zhou X, et al. Preoperative prediction of pelvic lymph nodes metastasis in earlystage cervical cancer using radiomics nomogram developed based on T2-weighted MRI and diffusion-weighted imaging. Eur J Radiol. 2019;114:128-35.

16. Poujade O, Morice P, Rouzier R, Madelenat P, Lecuru F, Muray JM, etal.Pathologic responserate after concomitantneoadjuvant radiotherapy and chemotherapy for adenocarcinoma of the uterine cervix: a retrospective multicentric study. Int J Gynecol Cancer. 2010;20:815-20.

17. Del Prete S, Caraglia M, Luce A, Montella L, Galizia G, Sperlongano $\mathrm{P}$, et al. Clinical and pathological factors predictive of response to neoadjuvant chemotherapy in breast cancer: a single center experience. Oncol Lett. 2019;18:3873-9.

18. Ouldamer L, Chas M, Arbion F, Body G, Cirier J, Ballester M, 
et al. Risk scoring system for predicting axillary response after neoadjuvant chemotherapy in initially node-positive women with breast cancer. Surg Oncol. 2018;27:158-65.

19. Kong TW, Kim J, Son JH, Kang SW, Paek J, Chun M, et al. Preoperative nomogram for prediction of microscopic parametrial infiltration in patients with FIGO stage IB cervical cancer treated with radical hysterectomy. Gynecol Oncol. 2016;142:109-14.

20. Resende U, Cabello C, Oliveira Botelho Ramalho S, Zeferino LC. Predictors of pathological complete response in women with clinical complete response to neoadjuvant chemotherapy in breast carcinoma. Oncology. 2018;95:229-38.

21. Hug V, Thames HD Jr, Polyzos A, Johnston D. Chemoresistance is not a cause of the apparent failure of adjuvant chemotherapy in postmenopausal women. Eur J Cancer Clin Oncol. 1988;24:713-8.

22. Hricak H, Lacey CG, Sandles LG, Chang YC, Winkler ML, Stern JL. Invasive cervical carcinoma: comparison of MR imaging and surgical findings. Radiology. 1988;166:623-31.

23. Park JS, Jeon EK, Chun SH, Won HS, Lee A, Hur SY, et al. ERCC1 (excision repair cross-complementation group 1) expression as a predictor for response of neoadjuvant chemotherapy for FIGO stage 2B uterine cervix cancer. Gynecol Oncol. 2011;120:275-9.

24. Rein DT, Kurbacher CM. The role of chemotherapy in invasive cancer of the cervix uteri: current standards and future prospects. Anticancer Drugs. 2001;12:787-95.

25. Jiapaer S, Furuta T, Tanaka S, Kitabayashi T, Nakada M. Potential strategies overcoming the temozolomide resistance for glioblastoma. Neurol Med Chir (Tokyo). 2018;58:405-21.

26. Fu J, Wang W, Wang Y, Liu C, Wang P. The role of squamous cell carcinoma antigen (SCC Ag) in outcome prediction after concurrent chemoradiotherapy and treatment decisions for patients with cervical cancer. Radiat Oncol. 2019;14:146.

27. Wang D, Lippard SJ. Cellular processing of platinum anticancer drugs. Nat Rev Drug Discov. 2005;4:307-20.

28. Weaver BA. How taxol/paclitaxel kills cancer cells. Mol Biol Cell. 2014;25:2677-81.

29. Lapresa M, Parma G, Portuesi R, Colombo N. Neoadjuvant chemotherapy in cervical cancer: an update. Expert Rev Anticancer Ther. 2015;15:1171-81. 